\title{
Global dynamics in a class of discrete-time epidemic models with disease courses
}

\author{
Lei Wang, Qianqian Cui and Zhidong Teng*
}

"Correspondence:

zhidong@xju.edu.cn

College of Mathematics and System

Sciences, Xinjiang University,

Urumqi, Xinjiang 830046, P.R. China

\begin{abstract}
In this paper, a class of discrete SIRS epidemic models with disease courses is studied. The basic reproduction number $R_{0}$ is computed. The main results on the permanence and extinction of the disease are established. That is, the disease-free equilibrium is globally attractive if $R_{0}<1$, and there exists a unique endemic equilibrium and the disease is also permanent if $R_{0}>1$.
\end{abstract}

MSC: 39A30; 92D30

Keywords: discrete epidemic model; disease course; basic reproduction number; permanence; global attractivity; extinction

\section{Introduction}

In recent years, more and more attention has been paid to the discrete-time epidemic models. There are several reasons for that. Firstly, since the statistic data about a disease is collected by day, week, month or year, it is more direct, more convenient and more accurate to describe the disease by using the discrete-time models than the continuoustime models; secondly, the discrete-time models have more wealthy dynamical behaviors; for example, the single-species discrete-time models have bifurcations, chaos and other more complex dynamical behaviors.

For a discrete-time epidemic model, we see that at the present time, the main research subjects are the computation of the basic reproduction number, the local and global stability of the disease-free equilibrium and endemic equilibrium, the extinction, persistence and permanence of the disease, and the bifurcations, chaos and more complex dynamical behaviors of the model, etc. Many important and interesting results can be found in articles [1-24] and the references cited therein.

In [4], the next generation matrix approach for calculating the basic reproduction number is summarized for discrete-time epidemic models. As applications, six disease models have been developed for the study of two emerging wildlife diseases: hantavirus in rodents and chytridiomycosis in amphibians. The comparison of deterministic and stochastic SIS and SIR type epidemic models in discrete time is discussed in [3]. In [8, 9], the discretetime SIS type epidemic models with periodic environment and with disease-induced mortality in density-dependence, respectively, are investigated. In [11], Izzo and Vecchio proposed an implicit nonlinear system of difference equations which represents the discrete counterpart of a large class of continuous models concerning the dynamics of an infection in an organism or in a host population. They also studied the limiting behavior of the discrete model and derived the basic reproduction number. Izzo, Muroya and Vecchio in

(c) 2013 Wang et al.; licensee Springer. This is an Open Access article distributed under the terms of the Creative Commons Attribution License (http://creativecommons.org/licenses/by/2.0), which permits unrestricted use, distribution, and reproduction in any medium, provided the original work is properly cited. 
[10] proved the globally asymptotic stability of the disease-free equilibrium for a general discrete-time model of population dynamics in the presence of an infection. For the discrete epidemic model with immigration of infectives, by adopting the means of the nonstandard discretization method from continuous epidemic, Jang and Eiaydi in [12] studied the globally asymptotic stability of the disease-free equilibrium, the locally asymptotic stability of the endemic equilibrium and the strong persistence of the susceptible class. Li and Wang in [15] discussed a SIS type discrete epidemic model with stage structure, where Beverton-Holt type and Richer type recruitment rates were considered, the global stability of the disease-free equilibrium and the dynamical complexity were investigated. In [17], the sufficient and necessary conditions for the global stability of the endemic equilibrium were established for a discrete epidemic model for the disease with immunity and latency in a heterogeneous host population. In [19], the bifurcations and chaos were proved in a discrete epidemic model with nonlinear incidence rates. The permanence and extinction are investigated in [20-22] for a class of discrete SIRS and SEIRS type epidemic models with time delays. In [24], a discrete mathematical model is formulated to investigate the transmission and control of SARS in China, where the basic reproductive number is obtained as a threshold to determine the asymptotic behavior of the model. Particularly, in [18] the authors studied the following class of disease epidemic models with the spread of an infection in a host population:

$$
\begin{aligned}
& y(n+1)=\alpha+(1-\beta) y(n)-\sum_{i=1}^{n} \psi_{i}(y(n+1)) x_{i}(n), \quad n \geq 0, \\
& x_{1}(n+1)=\left(1-a_{1}\right) x_{1}(n)+\phi_{1}(y(n+1)) x_{L}(n), \quad 1 \leq L \leq m, \\
& x_{i}(n+1)=\left(1-a_{i}\right) x_{i}(n)+\phi_{i}(y(n+1)) x_{i-1}(n), \quad i=2,3, \ldots, m .
\end{aligned}
$$

The global stability of disease-free equilibrium and endemic equilibrium and the permanence of the disease were obtained.

However, we know that many diseases have different disease courses, for example, tuberculosis, syphilis, AIDS, etc. Therefore, taking into account the epidemic models with disease courses is very important since disease pathogen bacteria with different course may have different reproduction and survival capacities, which indirectly influences the population growth. Under a different disease course, the transmission rate, the mortality and other vital parameters will be different [25-27].

Motivated by the above results, in this paper, we consider a class of discrete-time epidemic models with disease courses. We divide the total population into $m+2$ subgroups according to $m$ disease courses. Let $x(n)$ be the number of susceptible individuals at the $n$th generation, $y_{j}(n)(j=1,2, \ldots, m)$ denote the number of infectious individuals who are in the $j$ th course of a disease at $n$th generation, and let $z(t)$ denote the number of recovered individuals at the $n$th generation. We introduce the following assumptions.

(1) The susceptible $x$ has a constant input rate $\Lambda$ and a natural death rate $d$.

(2) The susceptible individuals of the $(n+1)$ th generation are only infected by the infectious individuals of the $n$th generation, and $\beta_{j}$ is the constant transmission coefficient of which the susceptible is infected by compartment $y_{j}$.

(3) After a susceptible individual contacts infectives and is infected, he/she will firstly enter compartment $y_{1}$, and then turn into compartments $y_{2}, y_{3}, \ldots$, finally into compartment $y_{m}$. 
(4) The infectious $y_{j}$ in the $j$ th disease course admits the constant natural death rate $d$, the constant death rate induced by disease $\alpha_{j}$, the constant recovery rate $\gamma_{j}$ and the constant transmit rate $\varepsilon_{j}$ from compartment $y_{i}$ to $y_{i+1}$.

(5) The recovered $z$ admits the constant natural death rate $d$, does not have permanent immunity, hence there is a constant transfer rate $\delta$ from the recovered class back to the susceptible class.

Base on the above assumptions, a class of discrete-time epidemic dynamical models with $m$ disease courses can be established as follows:

$$
\left\{\begin{array}{l}
x(n+1)=\Lambda+(1-d) x(n)+\delta z(n)-\sum_{i=1}^{m} \beta_{i} y_{i}(n) x(n+1), \\
y_{1}(n+1)=\left(1-p_{1}\right) y_{1}(n)+\sum_{i=1}^{m} \beta_{i} y_{i}(n) x(n+1), \\
y_{j}(n+1)=\left(1-p_{j}\right) y_{j}(n)+\varepsilon_{j-1} y_{j-1}(n), \quad j=2,3, \ldots, m, \\
z(n+1)=(1-\delta-d) z(n)+\sum_{i=1}^{m} \gamma_{i} y_{i}(n),
\end{array}\right.
$$

where $p_{i}=d+\varepsilon_{i}+\alpha_{i}+\gamma_{i}, i=1,2, \ldots, m-1, p_{m}=d+\alpha_{m}+\gamma_{m}$. For model (1), we always assume that the following basic hypotheses hold.

$\left(\mathrm{H}_{1}\right)$ For each $i=1,2, \ldots, m, d>0, \varepsilon_{i}>0, \alpha_{i} \geq 0, \beta_{i} \geq 0, \beta_{m}>0, \gamma_{i}>0, \delta \geq 0,0<p_{i}<1$ and $0<\delta+d<1$.

$\left(\mathrm{H}_{2}\right)$ Any solution of model (1) satisfies the following initial conditions:

$$
x(0)>0, \quad y_{1}(0)>0, \quad z(0)>0, \quad y_{j}(0)>0, \quad j=2,3, \ldots, m .
$$

Remark 1 For model (1), we can easily see that when $\beta_{1}>0$ then model (1) describes a discrete SIRS type epidemic model with disease courses, where $y_{i}(n)(i=1,2, \ldots, m)$ denotes the number of infectious individuals in the $i$ th course of the disease; and when $\beta_{1}=0$ then model (1) describes a discrete SEIRS type epidemic model with disease courses, where $y_{1}(n)$ is exposed and $y_{i}(n)(i=2,3, \ldots, m)$ denotes the number of infectious individuals in the $i$ th course of the disease.

Remark 2 In model (1), based on the above assumption (1), we know that the disease incidence term is denoted by $\sum_{i=1}^{m} \beta_{i} y_{i}(n) x(n+1)$. This makes $x(n+1)$, i.e., the susceptible number of the $(n+1)$ th generation, appear on both sides of the first equation. The reason for the above arguments is based on two considerations. On the one hand, it is influenced by the works given in $[11,18]$; on the other hand, for the sake of convenience for mathematical analysis, especially, the positivity of solutions in model (1).

In this paper, by developing the methods given in $[10,11,18]$, we will give the explicit expression of the basic reproduction number $R_{0}$. The criteria on the permanence and extinction of the disease will be established. That is, the disease-free equilibrium is globally attractive if $R_{0}<1$, and there exists a unique endemic equilibrium and the disease is also permanent if $R_{0}>1$.

This paper is organized as follows. In Section 2, as preliminaries we will give several lemmas which will be used in the proofs of the main results. In Section 3, the basic reproduction number is calculated, the existence on the disease-free equilibrium and endemic 
equilibrium is given and the theorem on the globally asymptotic stability of the diseasefree equilibrium is stated and proved. In Section 4, we will obtain the permanence of the disease. Conclusions are presented in the last section.

\section{Preliminaries}

Let $k$ be any positive integer, we denote $R_{+}^{k}=\left\{\left(x_{1}, x_{2}, \ldots, x_{k}\right): x_{i} \geq 0, i=1,2, \ldots, k\right\}$. For any sequence $\{f(n)\}$, we define

$$
\bar{f}=\limsup _{n \rightarrow \infty} f(n), \quad f_{-}=\liminf _{n \rightarrow \infty} f(n) .
$$

Firstly, on the positivity of solutions of model (1), we have the following result.

Lemma 1 For any solution $\left(x(n), y_{1}(n), y_{2}(n), \ldots, y_{m}(n), z(n)\right)$ of model (1), it holds that $x(n)>0, y_{j}(n)>0, z(n)>0(j=1,2, \ldots, m)$ for all $n>0$.

Proof From model (1), we can easily obtain

$$
\begin{aligned}
& x(1)=\frac{\Lambda+(1-d) x(0)+\delta z(0)}{1+\sum_{i=1}^{m} \beta_{i} y_{i}(0)}>0, \\
& y_{1}(1)=\left(1-p_{1}\right) y_{1}(0)+\sum_{i=1}^{m} \beta_{i} y_{i}(0) x(1)>0, \\
& y_{j}(1)=\left(1-p_{j}\right) y_{j}(0)+\varepsilon_{j-1} y_{j-1}(0)>0, \quad j=2,3, \ldots, m
\end{aligned}
$$

and

$$
z(1)=(1-\delta-d) z(0)+\sum_{i=1}^{m} \gamma_{i} y_{i}(0)>0
$$

Assume that $x(n)>0, y_{i}(n)>0(i=1,2, \ldots, m)$ and $z(n)>0$, then we further have

$$
\begin{aligned}
& x(n+1)=\frac{\Lambda+(1-d) x(n)+\delta z(n)}{1+\sum_{i=1}^{m} \beta_{i} y_{i}(n)}>0, \\
& y_{1}(n+1)=\left(1-p_{1}\right) y_{1}(n)+\sum_{i=1}^{m} \beta_{i} y_{i}(n) x(n+1)>0, \\
& y_{j}(n+1)=\left(1-p_{j}\right) y_{j}(n)+\varepsilon_{j-1} y_{j-1}(n)>0, \quad j=2,3, \ldots, m
\end{aligned}
$$

and

$$
z(n+1)=(1-\delta-d) z(n)+\sum_{i=1}^{m} \gamma_{i} y_{i}(n)>0
$$

Therefore, by using the induction, we get $x(n)>0, y_{j}(n)>0, z(n)>0$ for all $n>0$ and $j=1,2, \ldots, m$. This completes the proof.

Lemma 2 For any solution $\left(x(n), y_{1}(n), y_{2}(n), \ldots, y_{m}(n), z(n)\right)$ of model (1), it follows that

$$
\bar{x} \leq \frac{\Lambda}{d}, \quad \bar{y}_{j} \leq \frac{\Lambda}{d}, \quad \bar{z} \leq \frac{\Lambda}{d}, \quad j=1,2, \ldots, m .
$$


Proof Let

$$
N(n)=x(n)+\sum_{j=1}^{m} y_{j}(n)+z(n),
$$

then we have

$$
\begin{aligned}
N(n+1) & =\Lambda+(1-d) x(n)+\sum_{i=1}^{m}\left(1-d-\alpha_{i}\right) y_{i}(n)+(1-d) z(n) \\
& <\Lambda+(1-d) x(n)+\sum_{i=1}^{m}(1-d) y_{i}(n)+(1-d) z(n) \\
& =\Lambda+(1-d) N(n) .
\end{aligned}
$$

By using the induction, we can obtain the following inequality:

$$
N(n)<\frac{\Lambda}{d}\left[1-(1-d)^{n}\right]+(1-d)^{n} N(0),
$$

from which we have

$$
\bar{N}=\limsup _{n \rightarrow \infty} N(n) \leq \frac{\Lambda}{d} .
$$

From this, we finally have

$$
\bar{x} \leq \frac{\Lambda}{d}, \quad \bar{y}_{j} \leq \frac{\Lambda}{d}, \quad \bar{z} \leq \frac{\Lambda}{d}, \quad j=1,2, \ldots, m .
$$

This completes the proof.

On the weak permanence and permanence of the disease of model (1), we have the following definitions.

The disease in model (1) is said to be weak permanent (permanent) if there exists a constant $h>0$ such that, for any solution sequence $\left(x(n), y_{1}(n), y_{2}(n), \ldots, y_{m}(n), z(n)\right)$ of model (1), one has

$$
\limsup _{n \rightarrow \infty} y_{i}(n) \geq h\left(\liminf _{n \rightarrow \infty} y_{i}(n) \geq h\right), \quad i=1,2, \ldots, m .
$$

From Lemma 2, Theorem 1.1.3 and Theorem 1.10 given in [28], we can immediately obtain the following result.

Lemma 3 If the disease in model (1) is weak permanent, then it also is permanent.

Similar to Lemma 2.3 in [11] and Lemma 5 in [18], we have the following result.

Lemma 4 For any solution $\left(x(n), y_{1}(n), y_{2}(n), \ldots, y_{m}(n), z(n)\right)$ of model (1), the following inequalities hold:

$$
\begin{aligned}
& \frac{d \Lambda}{d^{2}+\sum_{i=1}^{m} \beta_{i} \Lambda} \leq \underline{x} \leq \bar{x} \leq \frac{\Lambda}{d}, \\
& \bar{y}_{j} \geq \varepsilon_{j-1} \bar{y}_{j-1}, \quad j=2,3, \ldots, m,
\end{aligned}
$$




$$
\begin{aligned}
& \prod_{i=1}^{j} \frac{\varepsilon_{i-1}}{p_{i}}\left(\sum_{i=1}^{m} \beta_{i} \underline{y}_{i} \underline{x}\right) \leq \underline{y}_{j} \leq \bar{y}_{j} \leq \prod_{i=1}^{j} \frac{\varepsilon_{i-1}}{p_{i}}\left(\sum_{i=1}^{m} \beta_{i} \bar{y}_{i} \bar{x}\right), \quad j=1,2, \ldots, m, \\
& \frac{1}{\delta+d} \sum_{i=1}^{m} \gamma_{i} \underline{y}_{i} \leq \underline{z} \leq \bar{z} \leq \frac{1}{\delta+d} \sum_{i=1}^{m} \gamma_{i} \bar{y}_{i} .
\end{aligned}
$$

Meanwhile, we also have

$$
D \underline{x} \sum_{i=1}^{m} \beta_{i} \underline{y}_{i} \leq \sum_{i=1}^{m} \beta_{i} \underline{y}_{i} \leq \sum_{i=1}^{m} \beta_{i} \bar{y}_{i} \leq D \bar{x} \sum_{i=1}^{m} \beta_{i} \bar{y}_{i}
$$

where

$$
D=\sum_{j=1}^{m} \frac{\varepsilon_{0} \varepsilon_{1} \cdots \varepsilon_{j-1} \beta_{j}}{p_{1} p_{2} \cdots p_{j}}, \quad \varepsilon_{0}=1
$$

Proof From model (1) and Lemmas 1 and 2, we easily have that

$$
\underline{x} \geq \Lambda+(1-d) \underline{x}-\sum_{i=1}^{m} \beta_{i} \bar{y}_{i} \underline{x} \geq \Lambda+(1-d) \underline{x}-\sum_{i=1}^{m} \frac{\Lambda}{d} \beta_{i} \underline{x}
$$

and then

$$
\underline{x} \geq \frac{d \Lambda}{d^{2}+\sum_{i=1}^{m} \beta_{i} \Lambda}>0 .
$$

From the third equation of model (1), we directly have

$$
y_{j}(n+1) \geq \varepsilon_{j-1} y_{j-1}(n), \quad j=2,3, \ldots, m, n=1,2, \ldots .
$$

Hence, we immediately obtain that $\bar{y}_{j} \geq \varepsilon_{j-1} \bar{y}_{j-1}$ for $j=2,3, \ldots, m$.

Considering the second equation of model (1), we can obtain the following inequality:

$$
\bar{y}_{1}=\limsup _{n \rightarrow \infty}\left\{\left(1-p_{1}\right) y_{1}(n)+\sum_{i=1}^{m} \beta_{i} y_{i}(n) x(n+1)\right\} \leq\left(1-p_{1}\right) \bar{y}_{1}+\sum_{i=1}^{m} \beta_{i} \bar{y}_{i} \bar{x}
$$

and

$$
\underline{y}_{1}=\liminf _{n \rightarrow \infty}\left\{\left(1-p_{1}\right) y_{1}(n)+\sum_{i=1}^{m} \beta_{i} y_{i}(n) x(n+1)\right\} \geq\left(1-p_{1}\right) \underline{y}_{1}+\sum_{i=1}^{m} \beta_{i} \underline{y}_{i} \underline{x} .
$$

Then we have

$$
\frac{1}{p_{1}} \sum_{i=1}^{m} \beta_{i} \underline{y}_{i} \underline{x} \leq \underline{y}_{1} \leq \bar{y}_{1} \leq \frac{1}{p_{1}} \sum_{i=1}^{m} \beta_{i} \bar{y}_{i} \bar{x} .
$$

Similarly, from model (1), we easily obtain

$$
\frac{\varepsilon_{j-1}}{p_{j}} y_{j-1} \leq \underline{y}_{j} \leq \bar{y}_{j} \leq \frac{\varepsilon_{j-1}}{p_{j}} \bar{y}_{j-1}, \quad j=2,3, \ldots, m
$$


and

$$
\frac{1}{\delta+d} \sum_{i=1}^{m} \gamma_{i} \underline{y}_{i} \leq \underline{z} \leq \bar{z} \leq \frac{1}{\delta+d} \sum_{i=1}^{m} \gamma_{i} \bar{y}_{i}
$$

Hence, from (7) and (8), it can be easily proved that

$$
\prod_{i=1}^{j} \frac{\varepsilon_{i-1}}{p_{i}}\left(\sum_{i=1}^{m} \beta_{i} \underline{y}_{i} \underline{\underline{x}}\right) \leq \underline{y}_{j} \leq \bar{y}_{j} \leq \prod_{i=1}^{j} \frac{\varepsilon_{i-1}}{p_{i}}\left(\sum_{i=1}^{m} \beta_{i} \bar{y}_{i} \bar{x}\right) .
$$

Hence, inequality (5) holds.

From inequalities (7), (8) and (9), it follows that

$$
\sum_{i=1}^{m} \beta_{i} \bar{y}_{i} \leq\left(\sum_{j=1}^{m} \frac{\varepsilon_{0} \varepsilon_{1} \cdots \varepsilon_{j-1} \beta_{j}}{p_{1} p_{2} \cdots p_{j}}\right)\left(\sum_{i=1}^{m} \beta_{i} \bar{y}_{i} \bar{x}\right)
$$

and

$$
\sum_{i=1}^{m} \beta_{i} \underline{y}_{i} \geq\left(\sum_{j=1}^{m} \frac{\varepsilon_{0} \varepsilon_{1} \cdots \varepsilon_{j-1} \beta_{j}}{p_{1} p_{2} \cdots p_{j}}\right)\left(\sum_{i=1}^{m} \beta_{i} \underline{y}_{i} \underline{x}\right) .
$$

Hence, inequality (6) holds. This completes the proof.

\section{Global attractivity of disease-free equilibrium}

Let the constant

$$
R_{0}=D \frac{\Lambda}{d}
$$

Firstly, on the existence of disease-free equilibrium and endemic equilibrium, we have the following result.

Theorem 1 (1) Model (1) always has a disease-free equilibrium $E_{0}\left(\frac{\Lambda}{d}, 0, \ldots, 0\right)$.

(2) When $R_{0}>1$, model (1) also has a unique endemic equilibrium $E^{*}\left(x^{*}, y_{1}^{*}, y_{2}^{*}, \ldots, y_{m}^{*}, z^{*}\right)$, where

$$
\begin{aligned}
& x^{*}=\frac{1}{D}, \quad y_{1}^{*}=\frac{R_{0}-1}{D d p_{1}\left(1-\frac{\delta}{\delta+d}\left(\sum_{i=1}^{m} \frac{\varepsilon_{0} \varepsilon_{1} \cdots \varepsilon_{l-1} \gamma_{l}}{p_{1} p_{2} \cdots p_{l}}\right)\right)}, \\
& y_{j}=\frac{\varepsilon_{1} \varepsilon_{2} \cdots \varepsilon_{j-1}}{p_{2} p_{3} \cdots p_{j}} y_{1}^{*}, \quad j=2,3, \ldots, m, \quad z^{*}=\frac{1}{\delta+d} \sum_{i=1}^{m} \gamma_{i} y_{i}^{*} .
\end{aligned}
$$

Proof The equilibrium of model (1) satisfies the following equations:

$$
\left\{\begin{array}{l}
x=\Lambda+(1-d) x+\delta z-\sum_{i=1}^{m} \beta_{i} y_{i} x \\
y_{1}=\left(1-p_{1}\right) y_{1}+\sum_{i=1}^{m} \beta_{i} y_{i} x \\
y_{j}=\left(1-p_{j}\right) y_{j}+\varepsilon_{j-1} y_{j-1}, \quad j=2,3, \ldots, m \\
z=(1-\delta-d) z+\sum_{i=1}^{m} \gamma_{i} y_{i} .
\end{array}\right.
$$


From the third equation to $(m+1)$-equation of $(11)$, we easily obtain

$$
y_{j}=\frac{\varepsilon_{1} \varepsilon_{2} \cdots \varepsilon_{j-1}}{p_{2} p_{3} \cdots p_{j}} y_{1}, \quad j=2,3, \ldots, m
$$

Substituting (12) into the second equation of (11), we further have

$$
y_{1}=\left(\frac{\beta_{1}}{p_{1}}+\frac{\varepsilon_{1} \beta_{2}}{p_{1} p_{2}}+\cdots+\frac{\varepsilon_{1} \varepsilon_{2} \cdots \varepsilon_{m-1} \beta_{m}}{p_{1} p_{2} \cdots p_{m}}\right) x y_{1}=D x y_{1} .
$$

Having solved this equality, we obtain that $y_{1}=0$ and $x=\frac{1}{D}$.

When $y_{1}=0$, then from (12) we have $y_{i}=0$ for $i=2,3, \ldots, m$. Further, from the first and the last equations of (11), we have $z=0$ and $x=\frac{\Lambda}{d}$. This shows that model (1) has a disease-free equilibrium $E_{0}\left(\frac{\Lambda}{d}, 0, \ldots, 0\right)$.

When $x=\frac{1}{D}$, then from the last equation of (11), we have

$$
z=\frac{1}{\delta+d} \sum_{i=1}^{m} \gamma_{i} y_{i}
$$

From the first equation of (11), we further obtain

$$
D\left(\Lambda+\frac{\delta}{\delta+d} \sum_{i=1}^{m} \gamma_{i} y_{i}\right)=d+\sum_{i=1}^{m} \beta_{i} y_{i}
$$

Substituting (12) into this equality, we further have

$$
\begin{aligned}
& D\left(\Lambda+\frac{\delta}{\delta+d}\left(\gamma_{1} y_{1}+\sum_{i \neq 1}^{m} \gamma_{i} \frac{\varepsilon_{1} \cdots \varepsilon_{i-1}}{p_{2} \cdots p_{i}} y_{1}\right)\right) \\
& =d+\beta_{1} y_{1}+\sum_{i \neq 1}^{m} \beta_{i} \frac{\varepsilon_{1} \cdots \varepsilon_{i-1}}{p_{2} \cdots p_{i}} y_{1} .
\end{aligned}
$$

Hence,

$$
D \Lambda-d=\left[\beta_{1}+\sum_{i \neq 1}^{m} \beta_{i} \frac{\varepsilon_{1} \cdots \varepsilon_{i-1}}{p_{2} \cdots p_{i}}-D \frac{\delta}{\delta+d}\left(\gamma_{1}+\sum_{i \neq 1}^{m} \gamma_{i} \frac{\varepsilon_{1} \cdots \varepsilon_{i-1}}{p_{2} \cdots p_{i}}\right)\right] y_{1} .
$$

Then, we further have

$$
\begin{aligned}
d\left(R_{0}-1\right) & =p_{1} y_{1}\left[\sum_{i=1}^{m} \beta_{i} \frac{\varepsilon_{0} \varepsilon_{1} \cdots \varepsilon_{i-1}}{p_{1} p_{2} \cdots p_{i}}-D \frac{\delta}{\delta+d} \sum_{i=1}^{m} \gamma_{i} \frac{\varepsilon_{0} \varepsilon_{1} \cdots \varepsilon_{i-1}}{p_{1} p_{2} \cdots p_{i}}\right] \\
& =p_{1} y_{1}\left[D-D \frac{\delta}{\delta+d} \sum_{i=1}^{m} \gamma_{i} \frac{\varepsilon_{0} \varepsilon_{1} \cdots \varepsilon_{i-1}}{p_{1} p_{2} \cdots p_{i}}\right] .
\end{aligned}
$$

Thus, we finally obtain

$$
y_{1}=\frac{d\left(R_{0}-1\right)}{D p_{1}\left(1-\frac{\delta}{\delta+d}\left(\sum_{j=1}^{m} \frac{\varepsilon_{0} \varepsilon_{1} \cdots \varepsilon_{j-1} \gamma_{j}}{p_{1} p_{2} \cdots p_{j}}\right)\right)} .
$$


Since $\varepsilon_{i}<p_{i}-\gamma_{i}$ for each $i=1,2, \ldots, m-1$ and $\gamma_{m}<p_{m}$, we have

$$
\begin{aligned}
& \gamma_{1} p_{2} \cdots p_{m}+\varepsilon_{1} \gamma_{2} p_{3} \cdots p_{m}+\cdots+\varepsilon_{1} \cdots \varepsilon_{m-2} \gamma_{m-1} p_{m}+\varepsilon_{1} \varepsilon_{2} \cdots \varepsilon_{m-1} \gamma_{m} \\
&<\gamma_{1} p_{2} \cdots p_{m}+\varepsilon_{1} \gamma_{2} p_{3} \cdots p_{m}+\cdots+\varepsilon_{1} \cdots \varepsilon_{m-2} \gamma_{m-1} p_{m} \\
& \quad+\left(p_{1}-\gamma_{1}\right) \cdots\left(p_{m-1}-\gamma_{m-1}\right) p_{m} \\
&=\left[\gamma_{1} p_{2} \cdots p_{m-1}+\varepsilon_{1} \gamma_{2} p_{3} \cdots p_{m-1}+\cdots+\varepsilon_{1} \cdots \varepsilon_{m-2} \gamma_{m-1}\right. \\
&\left.\quad+\left(p_{1}-\gamma_{1}\right) \cdots\left(p_{m-1}-\gamma_{m-1}\right)\right] p_{m} \\
&=\left[\gamma_{1} p_{2} \cdots p_{m-1}+\varepsilon_{1} \gamma_{2} p_{3} \cdots p_{m-1}+\cdots+\varepsilon_{1} \cdots \varepsilon_{m-3} \gamma_{m-2} p_{m-1}\right. \\
& \quad+\varepsilon_{1} \cdots \varepsilon_{m-2} \gamma_{m-1}+\left(p_{1}-\gamma_{1}\right) \cdots\left(p_{m-2}-\gamma_{m-2}\right) p_{m-1} \\
&\left.\quad-\left(p_{1}-\gamma_{1}\right) \cdots\left(p_{m-2}-\gamma_{m-2}\right) \gamma_{m-1}\right] p_{m} \\
&<[ {\left[\gamma_{1} p_{2} \cdots p_{m-1}+\varepsilon_{1} \gamma_{2} p_{3} \cdots p_{m-1}+\cdots+\varepsilon_{1} \cdots \varepsilon_{m-3} \gamma_{m-2} p_{m-1}\right.} \\
&\left.+\left(p_{1}-\gamma_{1}\right) \cdots\left(p_{m-1}-\gamma_{m-2}\right) p_{m-1}\right] p_{m} \\
&= {\left[\gamma_{1} p_{2} \cdots p_{m-2}+\varepsilon_{1} \gamma_{2} p_{3} \cdots p_{m-2}+\cdots+\varepsilon_{1} \cdots \varepsilon_{m-3} \gamma_{m-2}\right.} \\
&\left.+\left(p_{1}-\gamma_{1}\right) \cdots\left(p_{m-2}-\gamma_{m-2}\right)\right] p_{m-1} p_{m} \\
&< \cdots \\
&< {\left[\gamma_{1} p_{2}+\varepsilon_{1} \gamma_{2}+\left(p_{1}-\gamma_{1}\right)\left(p_{2}-\gamma_{2}\right)\right] p_{3} \cdots p_{m-1} p_{m} } \\
&= {\left[p_{1} p_{2}-\left(p_{1}-\gamma_{1}-\varepsilon_{1}\right) \gamma_{2}\right] p_{3} \cdots p_{m-1} p_{m} } \\
& \\
&
\end{aligned}
$$

Then we further obtain

$$
\begin{aligned}
\sum_{j=1}^{m} & \frac{\varepsilon_{0} \varepsilon_{1} \cdots \varepsilon_{j-1} \gamma_{j}}{p_{1} p_{2} \cdots p_{j}} \\
\quad= & \frac{\gamma_{1}}{p_{1}}+\frac{\varepsilon_{1} \gamma_{2}}{p_{1} p_{2}}+\cdots+\frac{\varepsilon_{1} \varepsilon_{2} \cdots \varepsilon_{m-1} \gamma_{m}}{p_{1} p_{2} \cdots p_{m}} \\
\quad= & \frac{\gamma_{1} p_{2} \cdots p_{m}+\varepsilon_{1} \gamma_{2} p_{3} \cdots p_{m}+\cdots+\varepsilon_{1} \cdots \varepsilon_{m-2} \gamma_{m-1} p_{m}+\varepsilon_{1} \varepsilon_{2} \cdots \varepsilon_{m-1} \gamma_{m}}{p_{1} p_{2} \cdots p_{m}} \\
& <\frac{p_{1} p_{2} \cdots p_{m}}{p_{1} p_{2} \cdots p_{m}}=1 .
\end{aligned}
$$

Hence, we can infer that

$$
\frac{\delta}{\delta+d}\left(\sum_{j=1}^{m} \frac{\varepsilon_{0} \varepsilon_{1} \cdots \varepsilon_{j-1} \gamma_{j}}{p_{1} p_{2} \cdots p_{j}}\right)<1
$$

Thus, from (14) we obtain that $y_{1}>0$ if and only if $R_{0}>1$. Further, from (12) we obtain $y_{i}>0$ for $i=2,3, \ldots, m$. Finally, from (13), we also have $z>0$. Therefore, we prove that model (1) has a unique endemic equilibrium $E^{*}$. This completes the proof. 
Remark 3 Obviously, we have

$$
R_{0}=\frac{\beta_{1} \Lambda}{p_{1} d}+\frac{\varepsilon_{1} \beta_{2} \Lambda}{p_{1} p_{2} d}+\cdots+\frac{\varepsilon_{1} \cdots \varepsilon_{m-1} \beta_{m} \Lambda}{p_{1} \cdots p_{m} d}
$$

The first term $\frac{\beta_{1} \Lambda}{p_{1} d}$ denotes the ultimate number of the susceptible at the end of the first disease course which is infected by an infectious individual of the first disease course. The second term $\frac{\varepsilon_{1} \beta_{2} \Lambda}{p_{1} p_{2} d}$ denotes the ultimate number of the susceptible at the end of the second disease course which is infected by an infectious individual of the second disease course. And lastly, the final term $\frac{\varepsilon_{1} \cdots \varepsilon_{m-1} \beta_{m} \Lambda}{p_{1} \cdots p_{m} d}$ denotes the ultimate number of the susceptible at the end of the $m$ th disease course which is infected by an infectious individual of the $m$ th disease course. We see that $R_{0}$ is the sum of these ultimate numbers. This shows that $R_{0}$ certainly is the basic reproduction number of model (1).

Theorem 2 If $R_{0}<1$, then the disease-free equilibrium $E_{0}$ of model (1) is globally attractive. That is, for any solution $\left(x(n), y_{1}(n), y_{2}(n), \ldots, y_{m}(n), z(n)\right)$ of model $(1)$, we have

$$
\lim _{n \rightarrow \infty} x(n)=\frac{\Lambda}{d}, \quad \lim _{n \rightarrow \infty} y_{j}(n)=0, \quad \lim _{n \rightarrow \infty} z(n)=0, \quad j=1,2, \ldots, m .
$$

Proof Since $R_{0}=D \frac{\Lambda}{d}<1$, then by inequality (6) in Lemma 4, we can obtain $\underline{y}_{j}=\bar{y}_{j}=0$ $(j=1,2, \ldots, m)$. In fact, if for some $j \in\{1,2, \ldots, m\}$ such that $\bar{y}_{j}>0$, then by inequality (4) in Lemma 4 , we can obtain $\bar{y}_{m}>0$. Hence, $\sum_{j=1}^{m} \beta_{j} \bar{y}_{j}>0$. From inequality (6) in Lemma 4 and $R_{0}<1$, we have

$$
\sum_{j=1}^{m} \beta_{j} \bar{y}_{j} \leq D \bar{x} \sum_{j=1}^{m} \beta_{j} \bar{y}_{j} \leq D \frac{\Lambda}{d} \sum_{j=1}^{m} \beta_{j} \bar{y}_{j}<\sum_{j=1}^{m} \beta_{j} \bar{y}_{j},
$$

which leads to a contradiction. Hence, $\lim _{n \rightarrow \infty} y_{j}(n)=0(j=1,2, \ldots, m)$. Finally, from the expression of $x(n)$ and $z(n)$ of model (1), we can infer that (15) holds. This completes the proof.

\section{Permanence of disease}

In this section, we mainly prove the permanence of model (1) when $R_{0}>1$. Firstly, we introduce several lemmas which will be used to study the permanence of model (1). Consider the following auxiliary system:

$$
\left\{\begin{array}{l}
u_{1}(n+1)=\left(1-p_{1}\right) u_{1}(n)+\sum_{i=1}^{m} \beta_{i} g(n+1) u_{i}(n), \\
u_{j}(n+1)=\left(1-p_{j}\right) u_{j}(n)+\varepsilon_{j-1} u_{j-1}(n), \quad j=2,3, \ldots, m, \\
u_{m+1}(n+1)=(1-\delta-d) y_{m+1}(n)+\sum_{i=1}^{m} \gamma_{i} u_{i}(n),
\end{array}\right.
$$

where $\{g(n)\}_{n=1}^{\infty}$ is a given non-negative bounded real sequence, and parameters $p_{i}, \beta_{i}, \gamma_{i}, \varepsilon_{j}$, $\delta$ and $d(i=1,2, \ldots, m, j=1,2, \ldots, m-1)$ are defined as in model (1). We have the following result.

Lemma 5 For any constants $\eta>0$ and $M>0$, there exist a constant $\xi=\xi(\eta)>0$ and an integer $T=T(M, \eta)>0$ such that for any initial time $n_{0} \in N_{+}$and initial value 
$\left(u_{10}, u_{20}, \ldots, u_{m+1,0}\right) \in R_{+}^{m+1}$ with $0<u_{i 0} \leq M(i=1,2, \ldots, m+1)$, if $u_{1}(n) \leq \xi$ for all $n \geq n_{0}$, then we have

$$
u_{i}(n) \leq \eta \quad \text { for all } n \geq n_{0}+T, i=1,2, \ldots, m+1,
$$

where $\left(u_{1}(n), u_{2}(n), \ldots, u_{m+1}(n)\right)$ is the solution of system (16) with the initial condition $\left(u_{1}\left(n_{0}\right), u_{2}\left(n_{0}\right), \ldots, u_{m+1}\left(n_{0}\right)\right)=\left(u_{10}, u_{20}, \ldots, u_{m+1,0}\right)$.

Proof Firstly, we consider the last equation of system (16)

$$
u_{m+1}(n+1)=(1-\delta-d) u_{m+1}(n)+\sum_{i=1}^{m} \gamma_{i} u_{i}(n)
$$

We can obtain that for any constants $\eta_{1}>0$ and $M>0$, there exist $\eta_{m+1}>0$ and $T_{m+1}>0$, with

$$
\eta_{m+1}=\frac{(\delta+d) \eta_{1}}{2 m}<\eta_{1}, \quad T_{m+1}>\frac{\ln \eta_{1}-\ln 2 M}{\ln (1-\delta-d)}
$$

such that for any initial time $n_{m+1}>0$ and initial value $0<u_{m+1} \leq M$, if $u_{i}(n) \leq \eta_{m+1}$ for all $n \geq n_{m+1}$ and $i=1,2, \ldots, m$, then we have

$$
\begin{aligned}
u_{m+1}(n) \leq & (1-\delta-d)^{n-n_{m+1}} u_{m+1}\left(n_{m+1}\right) \\
& +\left(\sum_{i=1}^{m} \gamma_{i} u_{i}(n)\right)\left(\sum_{j=1}^{\infty}(1-\delta-d)^{i}\right) \\
\leq & (1-\delta-d)^{n-n_{m+1}} M+\frac{\gamma m \eta_{m+1}}{\delta+d} \\
\leq & \eta_{1} \quad \text { for all } n \geq n_{m+1}+T_{m+1} .
\end{aligned}
$$

Consider the $m$ th equation of system (16)

$$
u_{m}(n+1)=\left(1-p_{m}\right) u_{m}(n)+\varepsilon_{m-1} u_{m-1}(n) .
$$

For the above constants $\eta_{m+1}>0$ and $M>0$, there exist a constant $\eta_{m}>0$ and an integer $T_{m}>0$, with

$$
\eta_{m}=p_{m} \eta_{m+1}<\eta_{m+1}, \quad T_{m}>\frac{\ln \left(1-\varepsilon_{m-1} \eta_{m+1}\right)-\ln M}{\ln \left(1-p_{m}\right)}
$$

such that for any initial time $n_{m}>0$ and initial value $0<u_{m}\left(n_{m}\right) \leq M$, if $u_{m-1}(n) \leq \eta_{m}$ for all $n \geq n_{m}$, then we have

$$
\begin{aligned}
u_{m}(n) & \leq\left(1-p_{m}\right)^{n-n_{m}} u_{m}\left(n_{m}\right)+\varepsilon_{m-1} \eta_{m} \sum_{i=1}^{\infty}\left(1-p_{m}\right)^{i} \\
& \leq\left(1-p_{m}\right)^{n-n_{m}} M+\frac{\varepsilon_{m-1} \eta_{m}}{p_{m}} \\
& \leq \eta_{m+1} \quad \text { for all } n \geq n_{m}+T_{m} .
\end{aligned}
$$


Further consider the $(m-1)$ th equation

$$
u_{m-1}(n+1)=\left(1-p_{m-1}\right) u_{m-1}(n)+\varepsilon_{m-2} u_{m-2}(n) .
$$

For the above constants $\eta_{m}$ and $M>0$, there exist a constant $\eta_{m-1}>0$ and an integer $T_{m-1}>0$, with

$$
\eta_{m-1}=p_{m-1} \eta_{m}<\eta_{m}, \quad T_{m-1}=\frac{\ln \left(1-\varepsilon_{m-2} \eta_{m}\right)-\ln M}{\ln \left(1-p_{m-1}\right)},
$$

such that for any initial time $n_{m-1}>0$ and initial value $0<u_{m-1}\left(n_{m-1}\right) \leq M$, if $u_{m-2}(n) \leq$ $\eta_{m-1}$ for all $n \geq n_{m-1}$, then we have

$$
\begin{aligned}
u_{m-1}(n) & \leq\left(1-p_{m-1}\right)^{n-n_{m-1}} u_{m-1}\left(n_{m-1}\right)+\varepsilon_{m-2} \eta_{m-1} \sum_{i=1}^{\infty}\left(1-p_{m-1}\right)^{i} \\
& \leq\left(1-p_{m-1}\right)^{n-n_{m-1}} M+\frac{\varepsilon_{m-2} \eta_{m-1}}{p_{m-1}} \\
& \leq \eta_{m} \quad \text { for all } n \geq n_{m-1}+T_{m-1} .
\end{aligned}
$$

Repeating the above process for $u_{m-2}(n), \ldots, u_{2}(n)$, respectively, finally we can obtain that for each $u_{i}(n)(i=2,3, \ldots, m-2)$ and for the above obtained constants $\eta_{i+1}>0$ and $M>0$, there exist a constant $\eta_{i}>0$ and an integer $T_{i}>0$, with

$$
\eta_{i}=p_{i} \eta_{i+1}<\eta_{i+1}, \quad T_{i}>\frac{\ln \left(1-\varepsilon_{1} \eta_{i+1}\right)-\ln M}{\ln \left(1-p_{i}\right)},
$$

such that for any initial time $n_{i}>0$ and $0<u_{i}\left(n_{i}\right) \leq M$, if $u_{i-1}(n) \leq \eta_{i}$ for all $n \geq n_{i}$, then we have

$$
\begin{aligned}
u_{i}(n) & \leq\left(1-p_{i}\right)^{n-n_{i}} u_{i}\left(n_{i}\right)+\varepsilon_{i-1} \eta_{i} \sum_{j=1}^{\infty}\left(1-p_{i}\right)^{j} \\
& \leq\left(1-p_{i}\right)^{n-n_{i}} M+\frac{\varepsilon_{i-1} \eta_{i}}{p_{i}} \\
& \leq \eta_{i+1} \quad \text { for all } n \geq n_{i}+T_{i} .
\end{aligned}
$$

Let $T=\sum_{i=2}^{m+1} T_{i}$. Then, from the above discussions, we obtain that for any initial time $n_{0}>0$ and initial value $0<u_{i}\left(n_{0}\right) \leq M(i=1,2, \ldots, m+1)$, if $u_{1}(n)<\eta_{2}$ for all $n \geq n_{0}$, then from (18) we have

$$
u_{2}(n)<\eta_{3}<\eta_{1} \quad \text { for all } n \geq n_{0}+T_{2} \text {. }
$$

We further have

$$
u_{3}(n)<\eta_{4}<\eta_{1} \quad \text { for all } n \geq n_{0}+T_{2}+T_{3} .
$$

Lastly, from (17) we have

$$
u_{m+1}(n)<\eta_{1} \quad \text { for all } n \geq n_{0}+T \text {. }
$$


This shows

$$
u_{i}(n)<\eta_{1} \quad \text { for all } n \geq n_{0}+T, i=1,2, \ldots, m+1 \text {. }
$$

This completes the proof.

We further consider the following equation:

$$
v(n+1)=\Lambda+(1-d) v(n)-\delta \eta-\sum_{i=1}^{m} \beta_{i} \eta\left(\frac{\Lambda}{d}+1\right)
$$

where the parameters are assumed to be as in system (1) and $0<\eta<1$. By calculating, we obtain that equation (19) has a positive equilibrium $v^{*}(\eta)$ with

$$
v^{*}(\eta)=\frac{\Lambda-\delta \eta-\sum_{i=1}^{m} \beta_{i} \eta\left(\frac{\Lambda}{d}+1\right)}{d} .
$$

Obviously, we have

$$
\lim _{\eta \rightarrow 0} v^{*}(\eta)=\frac{\Lambda}{d}
$$

Hence, there exists an $\eta_{0}>0$ such that when $0<\eta \leq \eta_{0}$, we have

$$
v^{*}(\eta) \leq \frac{\Lambda}{d}+1
$$

Therefore, we have the following result.

Lemma 6 For any constants $\varepsilon>0$ and $M>0$, there exists an integer $N_{0}=N_{0}(\varepsilon, M)>0$ such that for any initial $n_{0}>0$ and initial value $v_{0}$ with $0<v_{0} \leq M$, we have

$$
\left|v\left(n ; n_{0}, \eta\right)-v^{*}(\eta)\right|<\varepsilon \quad \text { for all } n \geq n_{0}+N_{0} \text { and } 0 \leq \eta \leq \eta_{0},
$$

where $v\left(n ; n_{0}, \eta\right)$ is the solution of equation (19) with the initial condition $v\left(n_{0}, \eta\right)=v_{0}$.

Proof For any solution $v\left(n ; n_{0}, \eta\right)$ of equation (19) with the initial condition $v\left(n_{0}, \eta\right)=v_{0}>$ 0 , we define a function

$$
V(n)=\left|v\left(n ; n_{0}, \eta\right)-v^{*}(\eta)\right|,
$$

then

$$
\begin{aligned}
V(n+1) & =\left|v\left(n+1 ; n_{0}, \eta\right)-v^{*}(\eta)\right| \\
& =(1-d)\left|v\left(n ; n_{0}, \eta\right)-v^{*}(\eta)\right| \\
& =(1-d) V(n) .
\end{aligned}
$$


From this, we easily see that for any constant $\varepsilon$, there exists an integer $N_{0}=N_{0}(\varepsilon, M)>0$ such that for any initial time $n_{0}>0$ and initial value $v_{0}$ with $0<v_{0} \leq M$, when $0<\eta \leq \eta_{0}$, then for all $n \geq n_{0}+N_{0}$, we have

$$
V(n) \leq(1-d)^{n-n_{0}}\left(M+\frac{\Lambda}{d}+1\right) \leq(1-d)^{N_{0}}\left(M+\frac{\Lambda}{d}+1\right)<\varepsilon .
$$

This shows that the conclusion of Lemma 6 holds. This completes the proof.

For any $x, y \in R^{m}$ with $x=\left(x_{1}, x_{2}, \ldots, x_{m}\right)$ and $y=\left(y_{1}, y_{2}, \ldots, y_{m}\right)$, if $x_{i} \leq y_{i}(i=1,2, \ldots, m)$, then we denote $x \leq y$.

Let $D \subset R^{m}$ and $f(n, u)=\left(f_{1}(n, u), f_{2}(n, u), \ldots, f_{m}(n, u)\right)$ be a function defined on $n \geq 0$ and $u \in D$. If for any $u_{1}, u_{2} \in D$ with $u_{1} \leq u_{2}$ we have

$$
f\left(n, u_{1}\right) \leq f\left(n, u_{2}\right) \text { for all } n \geq 0,
$$

then the function $f(n, u)$ is said to be non-decreasing for $u \in D$.

Lemma 7 (See [29]) Let the domain $D \subset R_{+}^{m}$ and the function $f(n, x)$ defined on $n \geq 0$ and $x \in D$ be non-decreasing for $x \in D$. If the sequence $\{x(n)\}_{n=1}^{\infty} \subset D$ for all $n \geq n_{0}$ satisfies $x(n+1) \geq f(n, y(n))(x(n+1) \leq f(n, y(n)))$, then we have

$$
x(n) \geq y(n) \quad(x(n) \leq y(n)) \quad \text { for all } n \geq n_{0},
$$

where $y(n)$ is the solution of the difference equation $y(n+1)=f(n, y(n))$ with initial value $x\left(n_{0}\right) \geq y\left(n_{0}\right)\left(x\left(n_{0}\right) \leq y\left(n_{0}\right)\right)$.

Now, we consider the following linear autonomous difference system:

$$
x(n+1)=A x(n),
$$

where $A$ is an $m \times m$ non-negative matrix and $x(n) \in R^{m}$. Then we have the following result.

Lemma 8 Let $r=r(A)$ be the spectral radius of matrix $A$, then the following conclusions hold.

(1) There exists an $m$-dimensional column vector $e=\left(e_{1}, e_{2}, \ldots, e_{m}\right)^{T}$ with $e_{i}>0$ $(i=1,2, \ldots, m)$ such that $x(n)=\lambda^{n} e$ is a solution of system $(22)$.

(2) For any $x_{0}=\left(x_{10}, x_{20}, \ldots, x_{m 0}\right)$ with $x_{i 0}>0(i=1,2, \ldots, m)$, there exist constants $a_{i}>0$ $(i=1,2)$ such that

$$
\lambda^{n-n_{0}} a_{1} e \leq x(n) \leq \lambda^{n-n_{0}} a_{2} e \quad \text { for all } n \geq n_{0},
$$

where $x(n)$ is the solution of system (22) satisfying the initial condition $x\left(n_{0}\right)=x_{0}$.

Proof (1) Since $\lambda$ is an eigenvalue of matrix $A$ and matrix $A$ is a non-negative matrix, we can obtain that there is a vector $e>0$ corresponding to the eigenvalue $\lambda$ such that

$$
A e=\lambda e .
$$


From $A^{n}=A A^{n-1}$, we have

$$
x(n)=A x(n-1)=A^{2} x(n-2)=\cdots=A^{n} x(0) .
$$

Suppose that $x(0)=e$, then

$$
x(n)=A^{n} e=A^{n-1} A e=\lambda A^{n-1} e \cdots=\lambda^{n-1} A e=\lambda^{n} e .
$$

Therefore, $x(n)=\lambda^{n} e$ is a solution of system (22).

(2) Let $b=\min _{1 \leq i \leq m}\left\{x_{i 0}\right\}, B=\max _{1 \leq i \leq m}\left\{x_{i 0}\right\}, c=\min _{1 \leq i \leq m}\left\{e_{i}\right\}$ and $C=\max _{1 \leq i \leq m}\left\{e_{i}\right\}$. Further let $a_{1}=b / C$ and $a_{2}=B / c$. Then we have $a_{1} e \leq x_{0} \leq a_{2} e$. Let $x_{1}(n)=a_{1} e \lambda^{n-n_{0}}$ and $x_{2}(n)=a_{2} e \lambda^{n-n_{0}}$. Then $x_{1}(n)$ and $x_{2}(n)$ are the solutions of system (22) with initial value $x_{1}\left(n_{0}\right)=a_{1} e$ and $x_{2}\left(n_{0}\right)=a_{2} e$, respectively. Then from Lemma 7 it follows that

$$
\lambda^{n-n_{0}} a_{1} e \leq x_{1}(n) \leq x(n) \leq x_{2}(n) \leq \lambda^{n-n_{0}} a_{2} e
$$

for all $n \geq n_{0}$.

Lemma 9 If $R_{0}>1$, then there exists a constant $h_{1}>0$ such that for any solution $\left(x(n), y_{1}(n), y_{2}(n), \ldots, y_{m}(n), z(n)\right)$ of model $(1)$, we have

$$
\limsup _{n \rightarrow \infty} y_{1}(n) \geq h_{1}
$$

Proof Since

$$
R_{0}=\frac{D \Lambda}{d}=\sum_{i=1}^{m} \frac{\varepsilon_{0} \varepsilon_{1} \cdots \varepsilon_{i-1} \beta_{i}}{p_{1} p_{2} \cdots p_{i}} \frac{\Lambda}{d}>1,
$$

then we can choose a constant $\delta_{0}\left(0<\delta_{0}<1\right)$ such that

$$
\sum_{i=1}^{m} \frac{\varepsilon_{0} \varepsilon_{1} \cdots \varepsilon_{i-1} \beta_{i}}{p_{1} p_{2} \cdots p_{i}}\left(\frac{\Lambda}{d}-\delta_{0}\right)>1
$$

Hence, we have

$$
p_{1} p_{2} \cdots p_{m}-\left(\beta_{1} p_{2} p_{3} \cdots p_{m}+\varepsilon_{1} \beta_{2} p_{3} \cdots p_{m}+\cdots+\varepsilon_{1} \cdots \varepsilon_{m-1} \beta_{m}\right)\left(\frac{\Lambda}{d}-\delta_{0}\right)<0
$$

Then, from (20) and (21), there exists an $\eta_{0}$ with $0<\eta_{0} \leq \delta_{0}$ such that

$$
\frac{\Lambda}{d}-\frac{\delta_{0}}{2} \leq v^{*}\left(\eta_{0}\right) \leq \frac{\Lambda}{d}+1
$$

Now, from Lemma 6, for above $\delta_{0}>0$, there exists an integer $N_{0}>0$ such that for any initial time $n_{0}>0$ and initial value $v_{0}$ with $0<v_{0} \leq M_{0}$, where $M_{0}=\frac{\Lambda}{d}+1$, we have

$$
v\left(n ; n_{0}, \eta_{0}\right)-v^{*}\left(\eta_{0}\right) \geq-\frac{\delta_{0}}{2} \quad \text { for all } n \geq n_{0}+N_{0}
$$


where $v\left(n ; n_{0}, \eta_{0}\right)$ is the solution of equation (19) with $\eta=\eta_{0}$ and the initial condition $v\left(n_{0}, \eta_{0}\right)=v_{0}$. Hence, from (25) and (26), we have

$$
v\left(n ; n_{0}, \eta_{0}\right) \geq \frac{\Lambda}{d}-\delta_{0} \quad \text { for all } n \geq n_{0}+N_{0} .
$$

Assume that $\left(x(n), y_{1}(n), \ldots, y_{m}(n), z(n)\right)$ is any positive solution of model (1) with the initial condition $\left(x\left(n_{0}\right), y_{1}\left(n_{0}\right), \ldots, y_{m}\left(n_{0}\right), z\left(n_{0}\right)\right)=\left(x_{0}, y_{10}, \ldots, y_{m 0}, z_{0}\right)$. Then, from Lemma 2 , for $\varepsilon=1$ there exists an integer $N_{1}>0$ such that when $n>N_{1}$, we have

$$
x(n) \leq \frac{\Lambda}{d}+1, \quad y_{j}(n) \leq \frac{\Lambda}{d}+1, \quad z(n) \leq \frac{\Lambda}{d}+1, \quad j=1,2, \ldots, m .
$$

Consider the following difference system:

$$
\left\{\begin{array}{l}
u_{1}(n+1)=\left(1-p_{1}\right) u_{1}(n)+\sum_{i=1}^{m} \beta_{i} x(n+1) u_{i}(n), \\
u_{j}(n+1)=\left(1-p_{j}\right) u_{j}(n)+\varepsilon_{j-1} u_{j-1}(n), \quad j=2,3, \ldots, m, \\
u_{m+1}(n+1)=(1-\delta-d) u_{m+1}(n)+\sum_{i=1}^{m} \gamma_{i} u_{i}(n),
\end{array}\right.
$$

where parameters $p_{i}, \beta_{i}, \varepsilon_{i}, \gamma_{i}, \delta$ and $d$ in system (29) are given as in model (1). Since $\left(x(n), y_{1}(n), \ldots, y_{m}(n), z(n)\right)$ is the solution of model $(1)$, then $\left(y_{1}(n), \ldots, y_{m}(n), z(n)\right)$ is the solution of system (29). From (28) and Lemma 5, for above $\eta_{0}>0$ and $M=\frac{\Lambda}{d}+1$, there exist a constant $\delta^{*}>0$ and an integer $N_{2}>0$ with $\delta^{*} \leq \eta_{0}$ such that for any initial time $n_{0} \geq N_{1}$ and initial value $\left(u_{10}, u_{20}, \ldots, u_{m+1,0}\right) \in R_{+0}^{m}$, if $u_{1}(n) \leq \delta^{*}$ for all $n \geq n_{0}$, then we have $u_{j}(n) \leq \eta_{0}$ for all $n \geq n_{0}+N_{2}$ and $j=1,2, \ldots, m+1$. Hence, if $y_{1}(n) \leq \delta^{*}$ for all $n \geq n_{0}$, then we have

$$
y_{j}(n) \leq \eta_{0}, \quad z(n) \leq \eta_{0} \quad \text { for all } n \geq n_{0}+N_{2}, j=1,2, \ldots, m .
$$

Now, we prove that if $R_{0}>1$ then

$$
\limsup _{n \rightarrow \infty} y_{1}(n) \geq \delta^{*}
$$

for any positive solution $\left(x(n), y_{1}(n), \ldots, y_{m}(n), z(n)\right)$ of model (1). Suppose that the conclusion is not true, then there exists a positive solution $\left(x(n), y_{1}(n), \ldots, y_{m}(n), z(n)\right)$ of model (1) such that $\lim _{\sup _{n \rightarrow \infty}} y_{1}(n)<\delta^{*}$. Hence, there exists an integer $N_{1}>0$ such that $y_{1}(n)<\delta^{*}$ for all $n \geq N_{1}$. From (30) we know that for any $n_{0} \geq N_{1}>0$, there exists an integer $N_{2}>0$ such that

$$
y_{j}(n) \leq \eta_{0} \quad \text { and } \quad z(n) \leq \eta_{0} \quad \text { for all } n \geq n_{0}+N_{2}, j=1,2, \ldots, m
$$

Then, from (28) and the first equation of model (1), we obtain

$$
x(n+1) \geq \Lambda+(1-d) x(n)-\delta \eta_{0}-\sum_{i=1}^{m} \beta_{i} \eta_{0}\left(\frac{\Lambda}{d}+1\right) .
$$


Since from Lemma 7 and (27), for any $n_{1} \geq n_{0}+N_{2}$, there exists an integer $N_{0}>0$ such that

$$
x(n) \geq \frac{\Lambda}{d}-\delta_{0} \quad \text { for all } n \geq n_{0}+N_{0}+N_{2},
$$

then replacing (32) into the second and the third equations of model (1), we have

$$
\left\{\begin{aligned}
y_{1}(n+1) & \geq\left(1-p_{1}\right) y_{1}(n)+\sum_{i=1}^{m} \beta_{i}\left(\frac{\Lambda}{d}-\delta_{0}\right) y_{i}(n) \\
y_{2}(n+1) & =\left(1-p_{2}\right) y_{2}(n)+\varepsilon_{1} y_{1}(n), \\
\cdots & \cdots \cdots \\
y_{m}(n+1) & =\left(1-p_{m}\right) y_{m}(n)+\varepsilon_{m-1} y_{m-1}(n) .
\end{aligned}\right.
$$

Next, consider the following auxiliary system:

$$
\left\{\begin{array}{c}
w_{1}(n+1)=\left(1-p_{1}\right) w_{1}(n)+\sum_{i=1}^{m} \beta_{i}\left(\frac{\Lambda}{d}-\delta_{0}\right) w_{i}(n) \\
w_{2}(n+1)=\left(1-p_{2}\right) w_{2}(n)+\varepsilon_{1} w_{1}(n) \\
\cdots \quad \ldots \quad \cdots \\
w_{m}(n+1)=\left(1-p_{m}\right) w_{m}(n)+\varepsilon_{m-1} w_{m-1}(n) .
\end{array}\right.
$$

Obviously, system (34) is a linear autonomous difference system and we can rewrite it as follows:

$$
w(n+1)=A w(n)
$$

where $w(n)=\left(w_{1}(n), w_{2}(n), \ldots, w_{m}(n)\right)^{T}$ and

$$
A=\left(\begin{array}{cccc}
1-p_{1}+\beta_{1}\left(\frac{\Lambda}{d}-\delta_{0}\right) & \beta_{2}\left(\frac{\Lambda}{d}-\delta_{0}\right) & \cdots & \beta_{m}\left(\frac{\Lambda}{d}-\delta_{0}\right) \\
\varepsilon_{1} & 1-p_{2} & \cdots & 0 \\
0 & \varepsilon_{2} & \cdots & 0 \\
\vdots & \vdots & \cdots & \vdots \\
0 & 0 & \cdots & 0 \\
0 & 0 & \cdots & 1-p_{m}
\end{array}\right)
$$

We have

$$
\begin{aligned}
f(\mu) & =|\mu E-A| \\
& =\left|\begin{array}{cccc}
\mu-1+p_{1}-\beta_{1}\left(\frac{\Lambda}{d}-\delta_{0}\right) & -\beta_{2}\left(\frac{\Lambda}{d}-\delta_{0}\right) & \cdots & -\beta_{m}\left(\frac{\Lambda}{d}-\delta_{0}\right) \\
-\varepsilon_{1} & \mu-1+p_{2} & \cdots & 0 \\
0 & -\varepsilon_{2} & \cdots & 0 \\
\vdots & \vdots & \cdots & \vdots \\
0 & 0 & \cdots & 0 \\
0 & 0 & \cdots & \mu-1+p_{m}
\end{array}\right| \\
& =\left(\mu-1+p_{1}-\beta_{1}\left(\frac{\Lambda}{d}-\delta_{0}\right)\right)\left(\mu-1+p_{2}\right) \cdots\left(\mu-1+p_{m-1}\right)
\end{aligned}
$$


Wang et al. Advances in Difference Equations 2013, 2013:57

Page 18 of 21

http://www.advancesindifferenceequations.com/content/2013/1/57

$$
\begin{aligned}
& \times\left(\mu-1+p_{m}\right)-\varepsilon_{1} \beta_{2}\left(\frac{\Lambda}{d}-\delta_{0}\right)\left(\mu-1+p_{3}\right) \cdots\left(\mu-1+p_{m-1}\right) \\
& \times\left(\mu-1+p_{m}\right)-\varepsilon_{1} \varepsilon_{2} \beta_{3}\left(\frac{\Lambda}{d}-\delta_{0}\right)\left(\mu-1+p_{4}\right) \cdots\left(\mu-1+p_{m-1}\right) \\
& \times\left(\mu-1+p_{m}\right)-\cdots-\varepsilon_{1} \varepsilon_{2} \cdots \varepsilon_{m-1} \beta_{m}\left(\frac{\Lambda}{d}-\delta_{0}\right) .
\end{aligned}
$$

From (24), we have

$$
\begin{aligned}
f(1)= & \left(p_{1}-\beta_{1}\left(\frac{\Lambda}{d}-\delta_{0}\right)\right) p_{2} p_{3} \cdots p_{m}-\varepsilon_{1} \beta_{2}\left(\frac{\Lambda}{d}-\delta_{0}\right) p_{3} \cdots p_{m} \\
& -\varepsilon_{1} \varepsilon_{2} \beta_{3}\left(\frac{\Lambda}{d}-\delta_{0}\right) p_{3} \cdots p_{m}-\cdots-\varepsilon_{1} \cdots \varepsilon_{m-2} \beta_{m-1}\left(\frac{\Lambda}{d}-\delta_{0}\right) p_{m} \\
& -\varepsilon_{1} \varepsilon_{2} \cdots \varepsilon_{m-1} \beta_{m}\left(\frac{\Lambda}{d}-\delta_{0}\right) \\
= & p_{1} p_{2} \cdots p_{m}-\left(\beta_{1} p_{2} p_{3} \cdots p_{m}+\varepsilon_{1} \beta_{2} p_{3} \cdots p_{m}+\cdots\right. \\
& \left.+\varepsilon_{1} \cdots \varepsilon_{m-1} \beta_{m}\right)\left(\frac{\Lambda}{d}-\delta_{0}\right) \\
< & 0
\end{aligned}
$$

and, for a constant $\beta>\max \left\{\beta_{1}, \beta_{2}, \ldots, \beta_{m}\right\}\left(\frac{\Lambda}{d}-\delta_{0}\right)$, we further have

$$
\begin{aligned}
& f(\beta+1)=\left(\beta+p_{1}-\beta_{1}\left(\frac{\Lambda}{d}-\delta_{0}\right)\right)\left(\beta+p_{2}\right)\left(\beta+p_{3}\right) \cdots\left(\beta+p_{m}\right) \\
& -\varepsilon_{1} \beta_{2}\left(\frac{\Lambda}{d}-\delta_{0}\right)\left(\beta+p_{3}\right) \cdots\left(\beta+p_{m}\right)-\cdots \\
& -\varepsilon_{1} \cdots \varepsilon_{m-2} \beta_{m-1}\left(\frac{\Lambda}{d}-\delta_{0}\right)\left(\beta+p_{m}\right) \\
& -\varepsilon_{1} \varepsilon_{2} \cdots \varepsilon_{m-1} \beta_{m}\left(\frac{\Lambda}{d}-\delta_{0}\right) \\
& =\left(\beta-\beta_{1}\left(\frac{\Lambda}{d}-\delta_{0}\right)\right)\left(\beta+p_{2}\right) \cdots\left(\beta+p_{m}\right) \\
& +p_{1} \beta\left(\beta+p_{3}\right) \cdots\left(\beta+p_{m}\right)+p_{1} p_{2} \beta\left(\beta+p_{4}\right) \cdots\left(\beta+p_{m}\right) \\
& +\cdots+p_{1} p_{2} \cdots p_{m-2} \beta\left(\beta+p_{m}\right)+p_{1} p_{2} \cdots p_{m-1}\left(\beta+p_{m}\right) \\
& -\varepsilon_{1} \beta_{2}\left(\frac{\Lambda}{d}-\delta_{0}\right)\left(\beta+p_{3}\right) \cdots\left(\beta+p_{m}\right)-\cdots \\
& -\varepsilon_{1} \cdots \varepsilon_{m-2} \beta_{m-1}\left(\frac{\Lambda}{d}-\delta_{0}\right)\left(\beta+p_{m}\right) \\
& -\varepsilon_{1} \varepsilon_{2} \cdots \varepsilon_{m-1} \beta_{m}\left(\frac{\Lambda}{d}-\delta_{0}\right) \\
& >\left(\beta-\beta_{1}\left(\frac{\Lambda}{d}-\delta_{0}\right)\right)\left(\beta+p_{2}\right) \cdots\left(\beta+p_{m}\right) \\
& +p_{1}\left(\beta-\beta_{2}\left(\frac{\Lambda}{d}-\delta_{0}\right)\right)\left(\beta+p_{3}\right) \cdots\left(\beta+p_{m}\right)
\end{aligned}
$$




$$
\begin{aligned}
& +p_{1} p_{2}\left(\beta-\beta_{3}\left(\frac{\Lambda}{d}-\delta_{0}\right)\right)\left(\beta+p_{4}\right) \cdots\left(\beta+p_{m}\right) \\
& +\cdots+p_{1} \cdots p_{m-2}\left(\beta-\beta_{m-1}\left(\frac{\Lambda}{d}-\delta_{0}\right)\right)\left(\beta+p_{m}\right) \\
& +p_{1} p_{2} \cdots p_{m-1}\left(\beta-\beta_{m}\left(\frac{\Lambda}{d}-\delta_{0}\right)\right) \\
& >0
\end{aligned}
$$

Then, from the intermediate value theorem, there exists a constant $\mu^{*} \in(1, \beta+1)$ such that $f\left(\mu^{*}\right)=0$. Therefore, we can obtain $\bar{\mu}=r(A)>1$. Let $w(n)$ be the solution of system (33) with the initial condition $w\left(N^{*}\right)=\left(y_{1}\left(N^{*}\right), y_{2}\left(N^{*}\right), \ldots, y_{m}\left(N^{*}\right)\right)$, where $N^{*}=n_{0}+N_{0}+N_{2}$. Then from (33), (34) and Lemma 7, we have

$$
y(n) \geq w(n) \quad \text { for all } n \geq N^{*}
$$

Further, from the second part of Lemma 8, there exists a constant $a_{1}>0$ such that

$$
w(n) \geq \bar{\mu}^{n-n_{0}-N_{0}-N_{2}} a_{1} e .
$$

Hence, we have

$$
y_{1}(n) \geq \bar{\mu}^{n-n_{0}-N_{0}-N_{2}} a_{1} e_{1} \geq \bar{\mu}^{n-n_{0}-N_{0}-N_{2}} a_{1} c .
$$

From this, we obtain

$$
\lim _{n \rightarrow \infty} y_{1}(n)=\infty
$$

which leads to a contradiction. This completes the proof.

Lastly, directly from Lemma 3, Lemma 4 and Lemma 9, we can obtain the following result on the permanence of model (1).

Theorem 3 If $R_{0}>1$, then the disease in model (1) is permanent.

Proof In fact, from Lemma 9, we obtain that for any positive solution $\left(x(n), y_{1}(n), \ldots, y_{m}(n)\right.$, $z(n))$ of model (1),

$$
\limsup _{n \rightarrow \infty} y_{1}(n) \geq h_{1}>0
$$

Then, from inequality (4) in Lemma 4, we further have

$$
\underset{n \rightarrow \infty}{\limsup } y_{j}(n) \geq \varepsilon_{2} \varepsilon_{3} \cdots \varepsilon_{j-1} \limsup _{n \rightarrow \infty} y_{1}(n) \geq \varepsilon_{2} \varepsilon_{3} \cdots \varepsilon_{j-1} h_{1}, \quad j=2,3, \ldots, m
$$

Finally, from the last equation of model (1), we have

$$
\limsup _{n \rightarrow \infty} z(n) \geq \sum_{i=1}^{m} \gamma_{i} \limsup _{n \rightarrow \infty} y_{i}(n) \geq \sum_{i=1}^{m} \gamma_{i} \varepsilon_{2} \varepsilon_{3} \cdots \varepsilon_{i-1} h_{1} .
$$

This completes the proof. 


\section{Conclusions}

In this paper, we study a class of discrete epidemic models with disease courses, that is, model (1). The basic reproduction number $R_{0}$ is calculated. It is shown that the global dynamics of model (1) is determined by the basic reproduction number $R_{0}$. If $R_{0}<1$, then we obtain that the disease-free equilibrium of model (1) is globally asymptotically stable. This shows that when $R_{0}<1$ the disease in model (1) is extinct. If $R_{0}>1$, then we obtain that the endemic equilibrium of model (1) exists and the disease is permanent. Clearly, our condition given in this paper is the threshold condition between the extinction and the permanence of the disease. Hence, our results obtained in this paper extend the results given in $[7,11,18]$ for the discrete epidemic models.

However, it is a pity that the case of the basic reproduction number $R_{0}=1$ is not discussed in this paper. From the results on the case $R_{0}=1$ obtained in $[17,18]$, we can guess that when $R_{0}=1$ then the disease-free equilibrium of model (1) is also globally asymptotically stable. This shows that when $R_{0}=1$ the disease in model (1) is also extinct. The other one which is not obtained in our this paper is the global stability of the endemic equilibrium of model (1). From the results on the global stability of the endemic equilibrium obtained in $[7,17,18]$, we can guess that when $R_{0}>1$ the endemic equilibrium of model (1) is globally stable. We will discuss these problems in our future work.

\section{Competing interests}

The authors declare that they have no competing interests.

\section{Authors' contributions}

The authors declare that the study was realized in collaboration with the same responsibility. All authors read and approved the final manuscript.

\section{Acknowledgements}

This work was supported by the National Natural Science Foundation of China (Grant Nos. 11271312, 11001235, 10901130), the China Postdoctoral Science Foundation (Grant Nos. 20110491750, 2012T50836) and the Natural Science Foundation of Xinjiang (Grant No. 2011211B08).

Received: 19 June 2012 Accepted: 21 February 2013 Published: 15 March 2013

\section{References}

1. Agarwal, RP, O'Regan, O, Wong, PJY: Dynamics of epidemics in homogeneous/heterogeneous populations and the spreading of multiple inter-related infectious diseases: constant-sign periodic solutions for the discrete model. Nonlinear Anal., Real World Appl. 8, 1040-1061 (2007)

2. Allen, LJS: Some discrete-time SI, SIR and SIS epidemic models. Math. Biosci. 124, 83-105 (1994)

3. Allen, LJS, Burgin, AM: Comparison of deterministic and stochastic SIS and SIR models in discrete time. Math. Biosci. $163,1-33(2000)$

4. Allen, LJS, Driessche, P: The basic reproduction number in some discrete-time epidemic models. J. Differ. Equ. Appl. 14, 1127-1147 (2008)

5. Allen, LJS, Lou, Y, Nevai, AL: Spatial patterns in a discrete-time SIS patch model. J. Math. Biol. 58, $339-375$ (2009)

6. Castillo-Chavez, C, Yakubu, AA: Discrete-time SIS models with complex dynamics. Nonlinear Anal. 47, 4753-4762 (2001)

7. Enatsu, Y, Nakata, Y: Global stability for a class of discrete SIR epidemic models. Math. Biosci. Eng. 7, 347-361 (2010)

8. Franke, LE, Yakubu, AA: Discrete-time SIS epidemic model in a seasonal environment. SIAM J. Appl. Math. 66, 1563-1587 (2006)

9. Franke, LE, Yakubu, AA: Disease-induced mortality in density-dependent discrete-time S-I-S epidemic models. J. Math. Biol. 57, 755-790 (2008)

10. Izzo, G, Muroya, Y, Vecchio, A: A general discrete time model of population dynamics in the presence of an infection. Discrete Dyn. Nat. Soc. 2009, Article ID 143019 (2009). doi:10.1155/2009/143019

11. Izzo, G, Vecchio, A: A discrete time version for models of population dynamics in the presence of an infection. J. Comput. Appl. Math. 210, 210-221 (2007)

12. Jang, S, Elaydi, SN: Difference equations from discretization of a continuous epidemic model with immigration of infectives. Technical report, V. 92, MTBI Cornell University (2004)

13. Li, J, Lou, J, Lou, M: Some discrete SI and SIS epidemic models. Appl. Math. Mech. 29, 113-119 (2008)

14. Li, J, Ma, Z, Brauer, F: Global analysis of discrete-time SI and SIS epidemic models. Math. Biosci. Eng. 4, 699-710 (2007)

15. Li, X, Wang, X: A discrete epidemic model with stage structure. Chaos Solitons Fractals 26, 947-958 (2005)

16. Oli, MK, Venkataraman, M, Klein, PA, Wendland, LD, Brown, MB: Population dynamics of infectious diseases: a discrete time model. Ecol. Model. 198, 183-194 (2006) 
17. Muroya, Y, Bellen, A, Enatsu, Y, Nakata, Y: Global stability for a discrete epidemic model for disease with immunity and latency spreading in a heterogeneous host population. Nonlinear Anal., Real World Appl. 13, 258-274 (2012)

18. Muroya, Y, Nakata, Y, Izzo, G, Vecchio, A: Permanence and global stability of a class of discrete epidemic models. Nonlinear Anal., Real World Appl. 12, 2105-2117 (2011)

19. Papaschinopoulos, G, Stefanidou, G, Papadopoulos, KB: On a modification of a discrete epidemic model. Comput. Math. Appl. 59, 3559-3569 (2010)

20. Sekiguchi, M: Permanence for some discrete epidemic models. Int. J. Biomath. 2, 443-461 (2009)

21. Sekiguchi, M: Permanence of a discrete SIRS epidemic model with time delays. Appl. Math. Lett. 23, 1280-1285 (2010)

22. Sekiguchi, M, Ishiwata, E: Global dynamics of a discretized SIRS epidemic model with time delay. J. Math. Anal. Appl. 371, 195-202 (2010)

23. Zhang, D, Shi, B: Oscillation and global asymptotic stability in a discrete epidemic model. J. Math. Anal. Appl. 278, 194-202 (2003)

24. Zhou, Y, Ma, M, Brauer, F: A discrete epidemic model for SARS transmission and control in China. Math. Comput. Model. 40, 1491-1506 (2004)

25. Andersen, BL, Kiecolt-Glaser, LK, Glaser, R: A biobehavioral model of cancer stress and disease course. Am. Psychol. 49(5), 389-404 (1994)

26. Kastbom, A, Strandberg, G, Lindroos, A, Skogh, T: Anti-CCP antibody test predicts the disease course during 3 years in early rheumatoid arthritis (The Swedish TIRA project). Ann. Rheum. Dis. 63, 1085-1089 (2004)

27. Vingsbo, C, Sahistrand, P, Brun, JG, Jonsson, R, Saxne, T, Holmdahl, R: A new model for rheumatoid arthritis with a chronic disease course influenced by both major histocompatibility complex and non-major histocompatibility complex genes. Am. J. Pathol. 149, 1675-1683 (1996)

28. Zhao, X: Dynamical Systems in Population Biology. Springer, New York (2003)

29. Wang, L, Wang, M: Ordinary Difference Equation. Xinjiang Univ. Press, Urumqi (1989)

doi:10.1186/1687-1847-2013-57

Cite this article as: Wang et al.: Global dynamics in a class of discrete-time epidemic models with disease courses. Advances in Difference Equations 2013 2013:57.

\section{Submit your manuscript to a SpringerOpen ${ }^{\circ}$ journal and benefit from:}

- Convenient online submission

- Rigorous peer review

- Immediate publication on acceptance

- Open access: articles freely available online

- High visibility within the field

- Retaining the copyright to your article 